
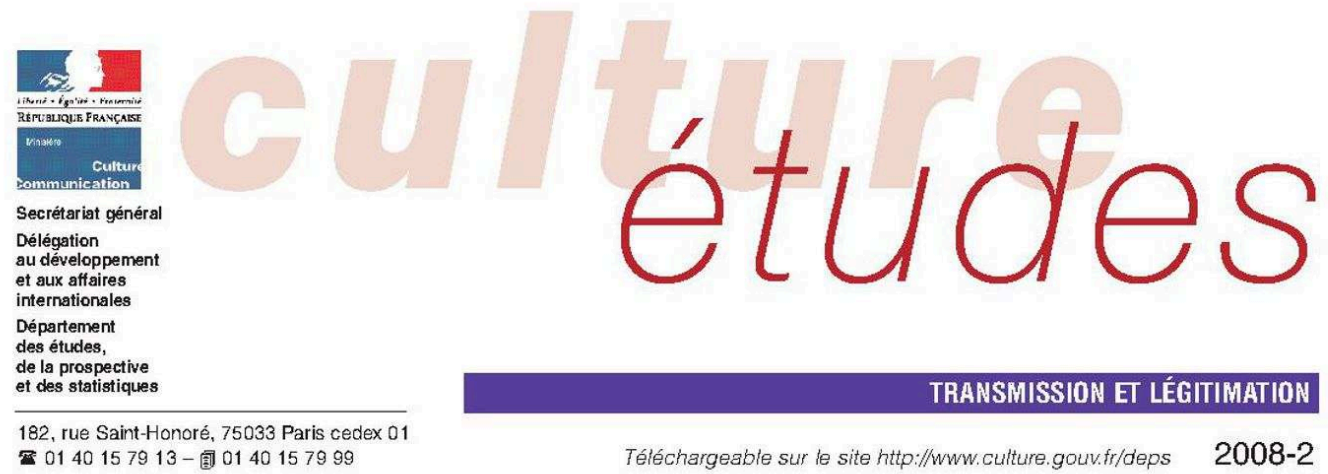

\title{
Les formations à l'administration et à la gestion de la culture : bilan et perspectives \\ Cécile MARTIN*
}

\section{Avant-propos}

La montée de l'emploi culturel depuis les années 1980 s'est accompagnée d'une professionnalisation croissante, en particulier à travers la multiplication de formations supérieures à l'administration et à la gestion de projets ou d'établissements culturels. À la rencontre de l'appétence des jeunes pour la culture, ces formations correspondent aussi à une forme de maturité économique des activités culturelles, sous l'effet de leur industrialisation et des contraintes budgétaires.

Pour autant, loffre de formation aux métiers de l'administration et de la gestion culturelles ne parait pas procéder d'une analyse de l'emploi culturel, des débouchés réels offerts, des perspectives de rémunération et de carrières. L'offre, principalement d'origine universitaire et généraliste, amplifie la forte densité du capital humain déjà caractéristique de l'emploi culturel, y compris dans des disciplines et pour des métiers où la composante culturelle ou artistique est faible : management, marketing, gestion, etc., et en dépit d'une différenciation par spécialités ou domaines d'activités.

Au moment où la plupart des États de l'Union européenne s'engagent dans des politiques en faveur de la croissance, de la compétitivité et de l'emploi, notamment à travers la dynamique économique culturelle, une réflexion sur la régulation et la répartition régionale de l'offre de formation au management culturel apparaît nécessaire et pourra se fonder sur les résultats de cette étude.
Comme dans bon nombre de pays européens, l'emploi culturel a connu en France une forte expansion en relation avec l'essor qu'a connu le secteur culturel depuis le début des années 1980. Cette expansion s'est accompagnée d'un mouvement de professionnalisation du champ culturel qui s'est appuyé sur une offre de formations destinées à favoriser l'adaptation des professionnels aux nécessités de la gestion, de l'administration et de la direction de projets d'entreprises ou d'établissements artistiques et culturels.

Attentif à l'émergence de ce secteur d'enseignement supérieur, le ministère de la Culture et de la Communication a soutenu un temps son développement de façon sélective. Dans les années 1990, la prolifération de ce type de filières a correspondu à des stratégies institutionnelles, professionnelles ou universitaires, partiellement maitrisées, répondant à la fois à une demande étudiante massive de formation compte tenu de l'attrait de la culture et aux besoins de responsables culturels en poste soucieux de valider leurs parcours et d'actualiser leurs compétences.

Du fait des transformations incessantes de l'environnement culturel à tous les niveaux - institutionnel, juridique, économique et technique - et de sa complexité croissante, ces formations doivent permettre à ceux qui les suivent d'acquérir des compétences sans cesse en évolution. De plus, l'extension du secteur culturel, mais aussi sa segmentation et la grande variété de ses métiers, engendrent un paysage très diversifié de l'offre de formation. Ainsi, le diplôme spécialisé devient de plus en plus le passage obligé pour faire carrière dans l'administration du secteur culturel.

\footnotetext{
* Texte réalisé à partir de la synthèse rédigé par Cécile Martin, directrice des études à l'Observatoire des politiques culturelles (OPC) avec la collaboration de Carole Alexandre, adjointe au chef du DEFEM/DDAs et de François Rouet, chargé d'études sur l'économie de la culture au
} DePS/DDal (ministère de la Culture et de la Communication). 


\section{Les formations à l'administration et à la gestion de la culture : bilan et perspectives}

Training in cultural administration and management: situation and outlook

\section{Cécile Martin (dir.)}

Éditeur : Département des études, de la prospective et des statistiques

Lieu d'édition : Paris

Année d'édition : 2008

Date de mise en ligne : 8 juillet 2015

Collection : Culture études

ISBN électronique : 9782111398825

\section{CboOks}

http://books.openedition.org

\section{Édition imprimée}

Date de publication : 1 avril 2008

Nombre de pages : 12

\section{Référence électronique}

MARTIN, Cécile (dir.). Les formations à l'administration et à la gestion de la culture : bilan et perspectives.

Nouvelle édition [en ligne]. Paris : Département des études, de la prospective et des statistiques, 2008 (généré le 25 avril 2021). Disponible sur Internet : <http://books.openedition.org/deps/286>. ISBN 9782111398825 

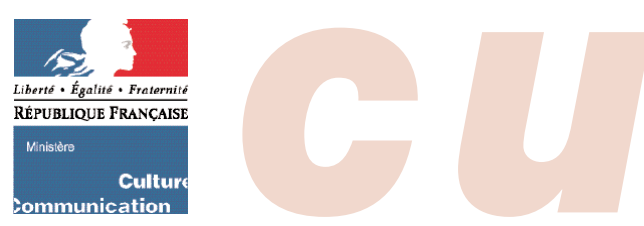

Secrétariat général

Délégation

au développement

et aux affaires

internationales

Département

des études,

de la prospective

et des statistiques

\section{Les formations à l'administration et à la gestion de la culture : bilan et perspectives}

Cécile MARTIN*

\section{Avant-propos}

La montée de l'emploi culturel depuis les années 1980 s'est accompagnée d'une professionnalisation croissante, en particulier à travers la multiplication de formations supérieures à l'administration et à la gestion de projets ou d'établissements culturels. À la rencontre de l'appétence des jeunes pour la culture, ces formations correspondent aussi à une forme de maturité économique des activités culturelles, sous l'effet de leur industrialisation et des contraintes budgétaires.

Pour autant, l'offre de formation aux métiers de l'administration et de la gestion culturelles ne paraît pas procéder d'une analyse de l'emploi culturel, des débouchés réels offerts, des perspectives de rémunération et de carrières. L'offre, principalement d'origine universitaire et généraliste, amplifie la forte densité du capital humain déjà caractéristique de l'emploi culturel, y compris dans des disciplines et pour des métiers où la composante culturelle ou artistique est faible : management, marketing, gestion, etc., et en dépit d'une différenciation par spécialités ou domaines d'activités.

Au moment où la plupart des États de l'Union européenne s'engagent dans des politiques en faveur de la croissance, de la compétitivité et de l'emploi, notamment à travers la dynamique économique culturelle, une réflexion sur la régulation et la répartition régionale de l'offre de formation au management culturel apparaît nécessaire et pourra se fonder sur les résultats de cette étude.
Comme dans bon nombre de pays européens, l'emploi culturel a connu en France une forte expansion en relation avec l'essor qu'a connu le secteur culturel depuis le début des années 1980. Cette expansion s'est accompagnée d'un mouvement de professionnalisation du champ culturel qui s'est appuyé sur une offre de formations destinées à favoriser l'adaptation des professionnels aux nécessités de la gestion, de l'administration et de la direction de projets d'entreprises ou d'établissements artistiques et culturels.

Attentif à l'émergence de ce secteur d'enseignement supérieur, le ministère de la Culture et de la Communication a soutenu un temps son développement de façon sélective. Dans les années 1990, la prolifération de ce type de filières a correspondu à des stratégies institutionnelles, professionnelles ou universitaires, partiellement maîtrisées, répondant à la fois à une demande étudiante massive de formation compte tenu de l'attrait de la culture et aux besoins de responsables culturels en poste soucieux de valider leurs parcours et d'actualiser leurs compétences.

Du fait des transformations incessantes de l'environnement culturel à tous les niveaux - institutionnel, juridique, économique et technique - et de sa complexité croissante, ces formations doivent permettre à ceux qui les suivent d'acquérir des compétences sans cesse en évolution. De plus, l'extension du secteur culturel, mais aussi sa segmentation et la grande variété de ses métiers, engendrent un paysage très diversifié de l'offre de formation. Ainsi, le diplôme spécialisé devient de plus en plus le passage obligé pour faire carrière dans l'administration du secteur culturel.

\footnotetext{
* Texte réalisé à partir de la synthèse rédigée par Cécile Martin, directrice des études à l'Observatoire des politiques culturelles (OPC) avec la collaboration de Carole Alexandre, adjointe au chef du DEFEM/DDAI et de François Rouet, chargé d'études sur l'économie de la culture au DEPS/DDAI (ministère de la Culture et de la Communication).
} 
Or, le développement sans précédent de ces formations n'a pas été accompagné d'une réflexion sur les outils nécessaires à la régulation du secteur et à l'adéquation de ces formations au marché de l'emploi ou la transformation des métiers, pas davantage sur l'offre de formation française dans le paysage européen.

Il s'agissait d'actualiser les connaissances sur l'offre de formation aux métiers de la gestion et de l'administration culturelles ${ }^{1}$. Il ne s'agit pas d'une évaluation des formations elles-mêmes, mais d'une collecte de données fiables permettant de dégager des éléments d'évaluation de ce champ et d'apprécier les modes d'articulation entre les formations existantes et le marché de l'emploi, exercice difficile au regard des données parcellaires sur l'emploi culturel relatif aux métiers d'administration et de gestion.

\section{Méthodologie de l'étude}

L'étude* s'est déroulée en trois phases:

- d'abord recensement le plus exhaustif possible des formations à la gestion et à l'administration de la culture de niveaux I, II et III, à partir des sites internet des établissements formateurs et d'une recherche documentaire complémentaire. Les données recueillies ont permis d'établir une première analyse quantitative ;

- ensuite l'objectif était de compléter l'analyse par des éléments qualitatifs et prospectifs; entretiens avec seize responsables de formations de niveau I dispensées par des universités, des écoles de commerce et des écoles dans le champ artistique et culturel ;

- enfin un séminaire de travail rassemblant une trentaine de responsables de formations de niveaux I et II, des représentants du ministère de la Culture, de collectivités territoriales, d'organismes impliqués dans la formation des professionnels. II a permis de mettre en débat les résultats et problématiques issus des deux phases précédentes. Une série de propositions pour l'avenir des formations a été formulée à l'issue du séminaire.

L'enquête a porté sur les formations supérieures diplômantes dans le domaine de l'administration et de la gestion de la culture, quels que soient l'organisateur ou le certificateur (universités, établissements consulaires ou privés...), le niveau d'études, la spécialisation principale, la périodicité de la formation, l'origine des étudiants (formation initiale ou continue)... Ont ainsi été prises en compte:

- les formations dispensées par des établissements publics de niveau bac + 2 (DU, DUT), bac + 3 (licences professionnelles), bac + 5 (masters professionnels);

- les formations dispensées par des établissements relevant du ministère de la Culture et agissant dans des champs disciplinaires spécifiques ;

- les formations dispensées par des établissements privés, de mêmes types et de mêmes niveaux que celles mentionnées ci-dessus, reconnues par le ministère de la Culture ou présentes dans le Répertoire national des certifications professionnelles (RNCC) www.cncp.gouv.fr;

- les formations dispensées par des écoles de commerce reconnues par la Conférence des grandes écoles (spécialisation culturelle en $2^{\mathrm{e}}$ ou $3^{\mathrm{e}}$ année, masters spécialisés délivrés par la $\left.\mathrm{CGE}^{\star \star}\right)$.

Les formations considérées pouvaient être spécialisées dans la gestion et l'administration de la culture ou dans une autre thématique (développement local, champs artistiques...) tout en intégrant un module long sur gestion et administration de la culture. Tous les secteurs artistiques et culturels ont été pris en compte dans la définition du champ de l'étude (spectacle vivant, patrimoine, musées, métiers d'art, métiers du livre, archives, bibliothèques, arts plastiques, cinéma, audiovisuel...).

Le champ "gestion et administration de la culture » ayant des frontières relativement floues et empiétant sur d'autres secteurs (professions de la médiation, de l'ingénierie culturelle, de la communication...), a donc été compris de façon assez extensive : en cas de doute sur certaines formations, celles-ci ont été intégrées à l'enquête.

Ont été exclues en revanche du champ de l'étude:

- les formations à la recherche (anciens DEA et masters recherche);

- les formations proposées sur une courte durée (inférieure à 1 semestre);

- les formations supérieures dispensées par des établissements privés, non reconnues par le ministère de la Culture ou non répertoriées au Répertoire national des certifications professionnelles (RNCP) au moment de l'étude; - les formations supérieures dispensées par des écoles de commerce consulaires mais non reconnues par la Conférence des grandes écoles;

- les formations post-recrutement de la fonction publique territoriale.

Les données recueillies dans cette enquête sont des données déclaratives, parfois incomplètes, provenant des sites internet des formations : elles n'ont pas été vérifiées systématiquement, dès lors qu'elles présentaient une cohérence interne suffisante.

\footnotetext{
*Étude commandée par le ministère de la Culture et de la Communication, DEPS, réalisée par l'Observatoire des politiques culturelles. Réalisation : AnneMarie AutISSIER, maître de conférences (Institut d'études européennes, université Paris 8), Garlonn BERTHOLOM, directrice de formation (École supérieure de commerce de Bretagne à Brest), Xavier DupuIs, professeur associé (université Paris-Dauphine), Cécile MARTIN, directrice des études (OPC), Mireille PONGY, chargée de recherche (CNRS, PACTE-IEP de Grenoble), Élisabeth RENAU, chargée de mission études et colloque (OPC), Philippe TEILLET, maître de conférences (IEP de Grenoble), Valentine RoY, chargée de mission (OPC), Jean-Pierre SAEZ, directeur (OPC). Coordination scientifique: Cécile MARTIN. Responsables du suivi au ministère de la Culture et de la Communication: Carole AleXANDRE (adjointe au chef du DEFEM/DDAI), François Rouet (chargé d'études sur l'économie de la culture au DEPS/DDAl).

Etude disponible en ligne sur www.culture gouv fr

** La CGE est une association qui regroupe des grandes écoles d'ingénieurs et 32 écoles de management, toutes à but non lucratif.
}

1. Une première investigation nationale avait en effet été réalisée par le ministère en 1992, suivie d'enquêtes sectorielles ou territorialisées, mais aucune donnée sur l'offre de formation dans ce secteur n'était disponible depuis cette date (voir Formation et emploi culturel. Les formations de $3^{e}$ cycle d'administrateurs culturels en question, Actes du séminaire du 2 juin 1992 à Grenoble, Ministère de la culture et de la francophonie, DDF/DEP, Université Pierre Mendès France Grenoble 2, Observatoire des politiques culturelles, mai 1993). 


\section{UNE OFFRE DE FORMATION}

\section{PLÉTHORIQUE ET CROISSANTE}

L'enquête a recensé 282 formations à la gestion et à l'administration de la culture ${ }^{2}$, qui se répartissent entre trois niveaux (graphique 1): 168 de niveau I (masters professionnels, masters spécialisés, autres diplômes de niveau I), 93 de niveau II (DU, DuT, licences professionnelles, licences disciplinaires, autres diplômes de niveau II) et 21 de niveau III (DU, DUT, DEUST, DEUG, autres diplômes de niveau III).

Les formations de niveau I sont majoritaires dans l'offre (presque 6 sur 10), ce qui se traduit par une offre pléthorique de masters professionnels $(55 \%$ des formations). Les masters en 2 ans se généralisent (29\% des formations) et correspondent à une adaptation à la réforme LMD.

Pour le niveau II (un tiers de l'offre), les licences professionnelles constituent $16 \%$ de l'offre globale de formation. Quant aux licences disciplinaires, plus souvent centrées sur des enseignements artistiques, elles sont peu présentes.

\section{Principales caractéristiques des formations}

\section{Ancienneté}

Parmi les 140 formations pour lesquelles la date de création a été repérée, les trois quarts ont été créées après 1990 et 32 \% depuis 2004. Les formations de niveau I ont été quant à elles pour moitié créées entre 1991 et 2003, et pour plus du tiers depuis 2004. Si l'application du processus de Bologne (encadré ci-dessous) joue un rôle dans cette inflation, ce n'est pas la seule raison puisque les dates d'habilitation des formations considérées dans le cadre de cette enquête sont celles de leurs premiers intitulés (DESS, licence professionnelle...).

Le processus de Bologne est issu d'une initiative intergouvernementale pour créer un Espace européen d'enseignement supérieur. Son principal instrument et le plus connu est la mise en place d'une structure commune des études supérieures articulée autour de trois niveaux: licence-bachelor, master, doctorat (LMD). S'y ajoutent la mise en place d'un cadre commun de références et de critères pour l'évaluation de la qualité des programmes et le développement d'un système de crédits facilitant la mobilité des étudiants.

\section{Graphique 1 - Répartition des formations à la gestion et à l'administration de la culture par niveau}

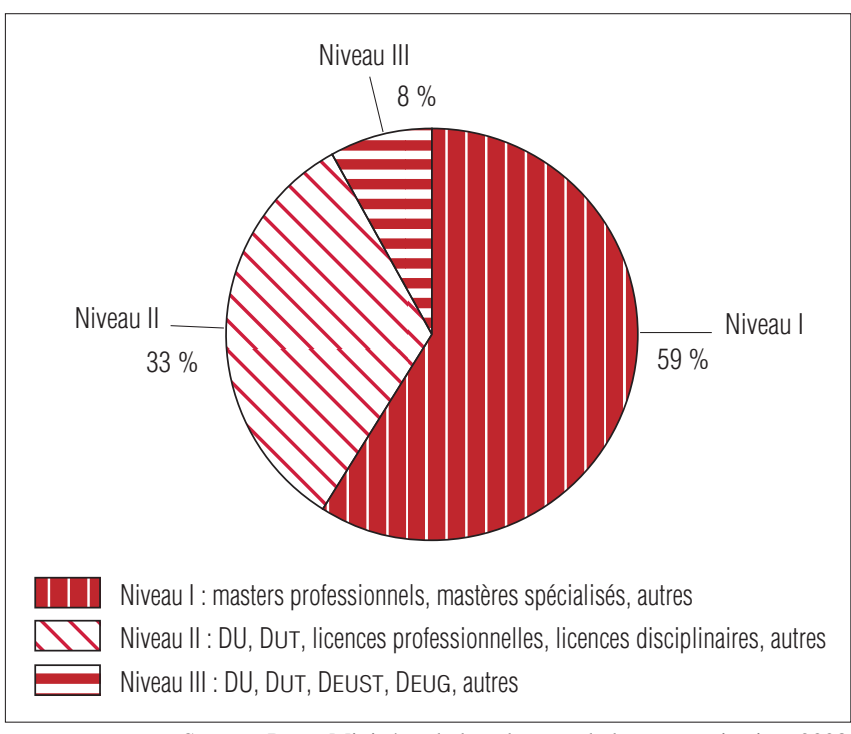

Source : DEPS, Ministère de la culture et de la communication, 2008

\section{Établissements formateurs}

L'offre de formation est à $88 \%$ certifiée par des établissements d'enseignement supérieur public relevant du ministère chargé de l'Enseignement supérieur (graphique 2). Cette offre se répartit principalement entre des établissements formateurs dépendant de l'Université, en particulier des facultés (plus de la moitié), des IuP et des IUT (un quart) qui bénéficient d'une autonomie plus grande au sein de l'Université et sont très présents dans ce secteur.

Les écoles de commerce accréditées par la Conférence des grandes écoles (CGE) proposent

\section{Graphique 2 - Répartition des établissements formateurs}

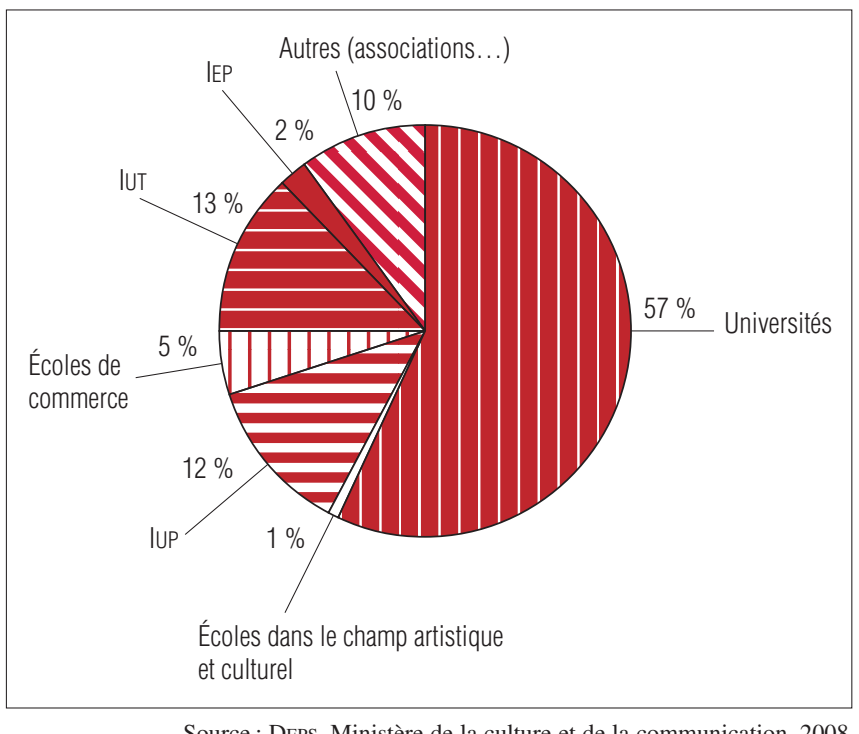

Source : DEPS, Ministère de la culture et de la communication, 2008

2. Les données présentées dans l'étude portent sur 275 formations pour lesquelles les informations recueillies étaient suffisamment précises pour permettre de les intégrer dans l'analyse statistique. 
également des formations : si certaines, comme la «majeure » proposée par le groupe HEC, existent depuis les années 1980, la plupart sont récentes ${ }^{3}$.

Quant aux écoles relevant du ministère de la Culture et de la Communication, qui proposent des formations en administration et gestion culturelles, il s'agit de formations spécialisées dans un secteur comme le cinéma (Fémis), la gestion et l'économie du patrimoine (École du Louvre), etc.

\section{Champs disciplinaires}

Au début des années 1980, les formations se sont très majoritairement réparties entre deux grands champs : arts, lettres et langues (36\%), sciences humaines et sociales et sciences politiques $(45 \%)$. À cet ancrage historique, se sont ajoutées, depuis les années 1990, les sciences de l'économie et de la gestion $(16 \%)$ ainsi que les sciences juridiques (3\%). Depuis, ces champs encore minoritaires apparaissent comme émergents, en lien notamment avec l'arrivée des écoles de commerce dans ce secteur, et les formations correspondantes témoignent d'une forte spécificité (graphique 3).

\section{Type de contenus}

Pour 7 formations sur 10, la spécialisation principale correspond aux métiers de l'administration, de la gestion et du management. Toutefois, les métiers de la médiation et de la communication semblent constituer une orientation spécifique au sein de la gestion et de l'administration de la culture (24\%), les formations spécialisées en ces domaines n'ont pas été recensées dans le cadre de cette étude. Quant aux formations tournées vers l'international et celles à dominante artistique, elles restent très marginales $(3 \%)$.

\section{Domaines d'application}

Le domaine d'application d'une formation - distinct du type de contenu qui renvoie aux métiers préparés - est soit généraliste, soit spécialisé dans un secteur.

\section{Généraliste ou sectoriel}

Parmi les formations recensées, $40 \%$ ont un domaine d'application généraliste, signifié par des intitulés du type «conduite et mise en œuvre de projets culturels ». Le secteur du patrimoine et celui des industries culturelles semblent bien dotés en formations spécialisées : respectivement $22 \%$ des formations pour patrimoine-archives-musées et $19 \%$ pour livre ${ }^{4}$-audiovisuel-musiques actuelles. Quant au domaine « développement culturel, territorial et touristique », il ne constitue que $5 \%$ des formations recensées (graphique 4).

\section{Secteur marchand ou non marchand, public ou privé}

Les établissements formateurs déclarent, pour 7 sur 10 d'entre eux, préparer leurs étudiants à travailler dans n'importe quel secteur, aussi bien marchand que non marchand, public que privé ; l'orientation spécifique « secteur marchand» ne représente que $13 \%$ des formations.

\section{Graphique 3 - Répartition des formations selon le champ disciplinaire}

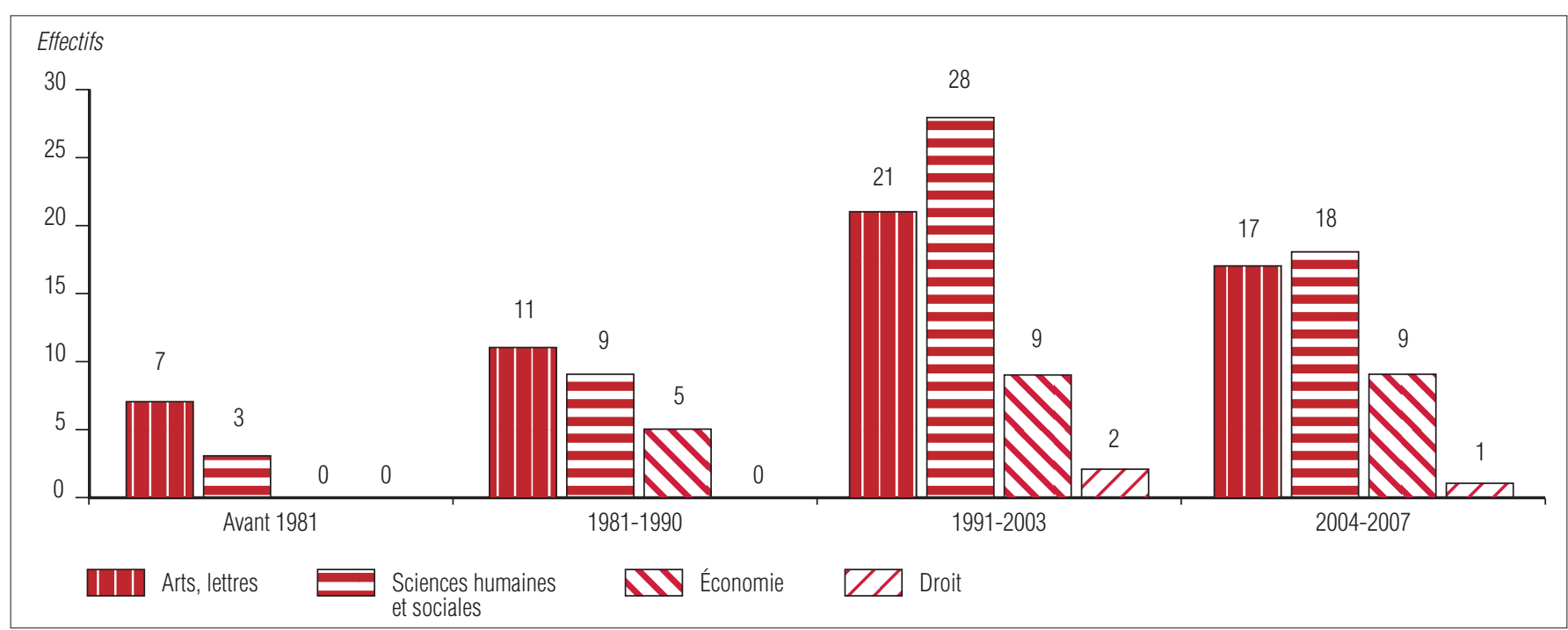

Source : DEPS, Ministère de la culture et de la communication, 2008

3. De nouvelles formations sont apparues en 2007, après la réalisation de cette enquête.

4. Notons que le domaine d'application «lecture publique » est faiblement représenté car il est amputé des formations qui préparent autant à la lecture publique qu'à l'édition, lesquelles ont été classées dans le secteur des industries culturelles. 
Graphique 4-Répartition des formations par domaine d'application

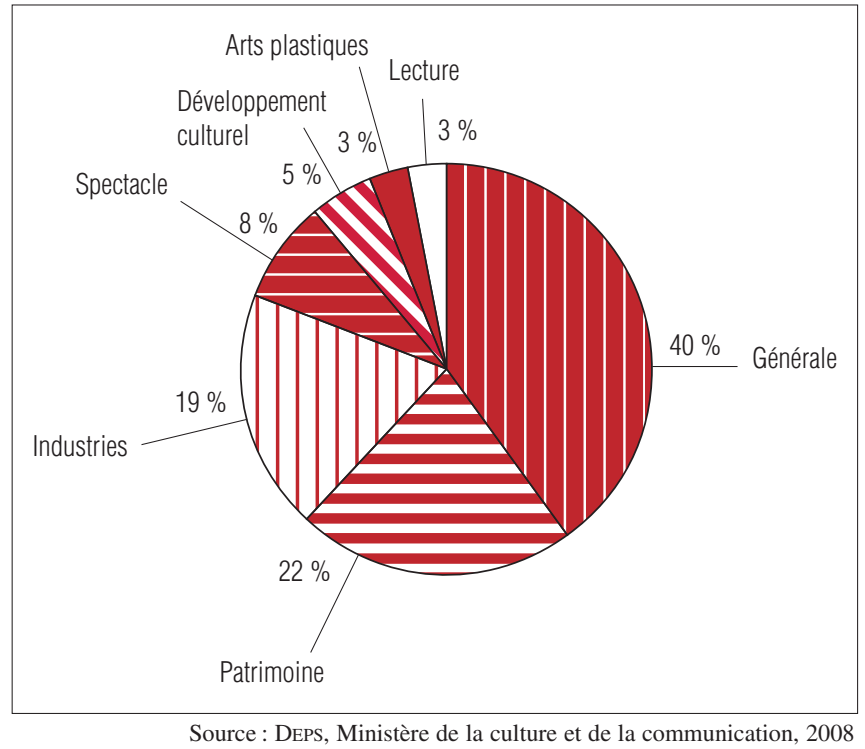

\section{Formation initiale/}

\section{formation pour professionnels}

Dans plus de 9 cas sur 10 (95\%), les diplômes sanctionnent uniquement une formation initiale. La tendance est en effet plus à l'ouverture de ces formations qu'à la création de filières spécifiques pour les professionnels : les formations destinées à ces derniers ne représentent que $5 \%$. De même, l'apprentissage reste très marginal. Quant aux dispositifs de validation des acquis de l'expérience (VAE) et de validation des acquis professionnels (VAP), si ces voies d'accès sont fréquemment mentionnées sur les sites internet des formations, aucune information quantitative précise n'est fournie en ce qui concerne l'ampleur de leur mise en œuvre.

\section{Effectifs d'étudiants par formation}

Selon les données recueillies - limitées puisque moins de 100 formations indiquent leurs effectifs $^{5}-$, le nombre d'étudiants accueillis par les établissements formateurs est en moyenne de 28 par an, ce qui témoigne de la relative sélectivité des formations. Les IuP présentent les effectifs les plus importants (43 étudiants en moyenne), les IEP et les écoles de commerce sont les établissements les plus sélectifs (moins de 20 étudiants par an).

Malgré ce recrutement moyen limité, pour les 95 formations dont l'effectif est connu, le nombre d'étudiants formés chaque année, tous niveaux confondus, peut être évalué à environ 2700 , chiffre qui permet d'estimer à 5000 le nombre total d'étudiants sortant chaque année de ces formations.

\section{Des formations surtout à temps plein...}

La quasi-totalité des formations $(98,5 \%)$ dispensent leurs enseignements à temps plein et seulement $1,5 \%$ par alternance ${ }^{6}$. Certaines proposent une organisation d'enseignement sur une période de temps plus longue comprenant à la fois du temps plein et de l'alternance. Le système de la semestrialisation a été adopté par la plupart d'entre elles, avec une organisation sur 2 à 4 semestres qui correspond respectivement à l'importance des licences professionnelles ( 1 an) et des masters (2 ans).

\section{... avec un nombre d'heures variant de 300 à 2500 heures}

Le volume horaire global, très variable, est en moyenne de 1000 heures $^{7}$. Les différences majeures ne se situent pas entre établissements certificateurs, mais entre diplômes délivrés où l'on constate de fortes disparités. Ainsi les formations en deux ans délivrant un Dut et les licences disciplinaires en trois ans proposent majoritairement un volume horaire élevé (entre 1000 et 2500 heures). Les troisièmes années des licences disciplinaires, licences professionnelles et masters 2 , en un an, présentent des volumes horaires à peu près équivalents (entre 500 et 1500 heures). Les masters en deux ans ont un volume horaire très variable, allant de 500 à plus de 2000 heures. Enfin, les mastères spécialisés, délivrés par les écoles supérieures de commerce, proposent pour la plupart de 1000 à 1500 heures de formation.

\section{Types d'enseignements}

Les formations à l'administration et à la gestion de la culture proposent plusieurs types d'enseignement : culture générale, enseignements théoriques explicitant les enjeux et enseignements techniques décrivant les outils appliqués à la sphère culturelle, enseignements artistiques et enseignements à orientation internationale.

Globalement, on constate peu de différences entre les types d'établissements formateurs. Toutefois, certaines particularités peuvent être relevées : - les facultés ne délivrent pas plus d'enseignements de culture générale ou théorique que les autres

5. Les effectifs peuvent également ne pas être indiqués lorsque le nombre d'étudiants n'est pas limité $a$ priori.

6. Données sur 269 formations répondantes.

7. Données sur 209 formations répondantes. 
types d'établissement, mais davantage d'enseignements artistiques et moins d'enseignements techniques spécialisés sur le domaine culturel ;

- les IUP et IUT, qui délivrent majoritairement des diplômes de niveaux II et III, insistent sur les enseignements de culture générale et théorique en matière culturelle, à parts égales avec les enseignements techniques. Les IUP se distinguent également par leur attention particulière pour les cours de langues. On constate donc une baisse relative du volume des enseignements de culture générale et des enseignements «théoriques spécialisés culture » à mesure que le niveau du diplôme s'élève.

Preuve de l'importance de la spécialisation, les enseignements de culture générale sont relativement marginaux, quel que soit le domaine d'application de la formation, général ou sectoriel. Les enseignements tournés vers l'international sont également peu valorisés : $38 \%$ des formations les ignorent et seules 22 formations proposent des modules conséquents (entre 100 et 299 heures), ce qui peut surprendre dans le contexte actuel d'européanisation et de mondialisation des échanges culturels.

Les enseignements théoriques et techniques spécialisés sur le secteur culturel, ainsi que ceux consacrés à la dimension artistique, diffèrent en volume selon le domaine d'application des formations. Les premiers sont absents dans $27 \%$ des formations et assez peu représentés dans $32 \%$ des cas. Ils sont davantage présents dans les formations à visée généraliste, ainsi que dans les formations spécialisées sur le spectacle vivant. Les enseignements consacrés aux aspects techniques représentent des volumes d'heures très importants dans la plupart des formations. C'est encore plus net dans le cas des formations tournées vers le patrimoine et les industries culturelles : ils représentent entre 100 et 299 heures pour $62 \%$ d'entre elles, voire de 300 à 499 heures pour $20 \%$.

Ceci souligne le caractère essentiellement technique des formations à l'administration et à la gestion de la culture, même si la dimension artistique des formations n'est pas oubliée (71\% des formations proposent des cours d'histoire de l'art, d'esthétique ou l'approfondissement d'une filière artistique). Cette dimension artistique, plus marquée dans les filières consacrées au domaine du spectacle vivant, est totalement absente d'un tiers des formations généralistes et de celles qui sont tournées vers les industries culturelles.

\section{Cours magistraux et stages}

Dans plus de $90 \%$ des cas, les formations proposent des enseignements classiques sous forme de cours magistraux - le plus souvent pour des volumes horaires de 300 à 700 heures - et des stages dont la durée s'échelonne en général de 2 à 6 mois (dans $18 \%$ des cas, la durée peut dépasser 6 mois). À l'occasion de ces stages, un rapport est demandé par $63 \%$ des formations, montrant en cela l'importance du stage dans ce type de parcours professionnalisants. Quant au montage de projet en «grandeur réelle» utilisé comme outil pédagogique, peu de formations y recourent et son utilisation reste encore relativement marginale.

\section{Une implantation sur l'ensemble du territoire...}

Les formations à l'administration et à la gestion culturelles sont implantées sur l'ensemble du territoire métropolitain, avec une surreprésentation, notamment pour les formations de niveau I, dans les régions Île-de-France et Rhône-Alpes ${ }^{8}$ (respectivement $27,3 \%$ et $13,5 \%$ des formations tous niveaux confondus). D'autres régions sont également bien pourvues - Provence-Alpes-Côte d'Azur $(7,3 \%)$ et ouest de la France avec Pays de la Loire $(7,6 \%)$, puis Bretagne et Aquitaine $(5,5 \%$ chacune). Ces régions présentent une offre équilibrée entre les formations de niveaux I et II, le niveau III restant plus marginal sur l'ensemble du territoire. Le nord du territoire, à l'exception notable du NordPas-de-Calais $(5,8 \%)$, et le centre de la France sont moins bien dotés (une seule formation en Limousin, deux en Picardie...).

\section{... mais une offre spécialisée inégalement répartie}

Hors Limousin et Picardie, toutes les régions proposent au moins une formation dédiée spécifiquement à l'administration et à la gestion de la culture. Les formations orientées vers le patrimoine, les archives, les musées sont les plus fréquemment proposées, et tout de suite derrière les formations spécialisées dans les industries culturelles. Aucune région ne se limite à des formations spécialisées dans un seul domaine d'application. On peut néanmoins relever quelques spécialisations en Île-deFrance (industries culturelles) et Rhône-Alpes (spectacle vivant). Ces régions, ainsi que la Bretagne, ont cependant une offre particulièrement complète en termes de secteurs couverts (du patrimoine aux industries culturelles...).

8. La surreprésentation constatée dans ces deux régions se retrouve dans de nombreuses enquêtes culturelles. 
Un fort ancrage professionnel et territorial pour des formations de niveau I (master ou équivalent), majoritairement universitaires ${ }^{9}$

\section{Origine et structuration}

Les formations nées dans le contexte des années 1980 résultaient d'un double constat: besoin de professionnalisation du secteur culturel et absence de formations appropriées. Ces formations ont le plus souvent été portées par des personnalités fortes, des universitaires très impliqués dans les milieux culturels. Récentes ou non, ces formations procèdent aussi fréquemment d'une analyse en termes d'aménagement du territoire, ce qui s'est traduit parfois par un soutien des collectivités territoriales (Arles, La Rochelle, Limoges...). Plus rarement, elles émanent d'une demande des milieux professionnels (master «musiques actuelles » d'Angers).

Si la plupart des formations de niveau I se déclarent généralistes ou proposent une orientation large, certaines sont au contraire très spécialisées sur un champ culturel, voire sur des métiers spécifiques.

L'inflation des formations à l'administration et à la gestion de la culture, en particulier des formations de niveau I, se traduit par une concurrence manifeste entre de nombreux cursus, qu'il s'agisse du recrutement des étudiants ou de leur insertion sur le marché de l'emploi.

\section{Les partenaires institutionnels}

Très présent aux côtés des premiers Dess dans les années 1990, le ministère de la Culture et de la Communication s'est progressivement éloigné de ces formations toujours plus nombreuses (en dehors de celles dispensées par des établissements placés sous sa tutelle), leur gestion relevant du ministère de l'Enseignement supérieur, dont dépendent les demandes d'habilitation.

Les collectivités territoriales se sont en revanche impliquées de façon croissante auprès des formations à l'administration et à la gestion culturelles, allant parfois jusqu'à signer des conventions avec elles (Grenoble, Arles, Mulhouse, Nantes, La Rochelle, conseil régional de Bretagne, conseil général du Finistère ou encore celui du Haut-Rhin...). Dans certains cas, des relations régulières ont également été mises en place avec des institutions culturelles locales ou nationales implantées dans les villes ou régions où sont proposées ces formations.

\section{Des étudiants aux profils très variés}

Les critères et les modalités de recrutement (dossier, épreuves, jury...) varient considérablement d'une formation à l'autre, et les prérequis (pratique des langues étrangères, connaissances en histoire de l'art...) ne sont pas toujours affichés précisément. Le recrutement est volontairement éclectique dans certaines formations (étudiants possédant une formation préalable en gestion, sciences humaines, sciences politiques...), dans d'autres cas nettement plus spécialisé (Fémis, École du Louvre, master « patrimoine et musées » de l'université de HauteAlsace...).

Peu de formations proposent des cursus spécifiquement destinés aux professionnels, mais il est fréquent que l'on compte un quart à un tiers de salariés en formation initiale. Les modalités de mise en œuvre vont des cursus communs aux cursus partagés, voire parallèles, entre formation continue et formation initiale. Certains masters sont ainsi particulièrement organisés en fonction de la formation continue, avec des rythmes d'enseignement et des modes d'évaluation spécifiques.

En formation continue, le diplôme est souvent un outil de promotion sociale pour des personnes qui n'ont pas bénéficié d'un cursus universitaire. Si la VAP est pratiquée sans réserve, la VAE est également évoquée comme un outil de promotion sociale pour les professionnels mais n'est pas décrite concrètement même si des expériences sont en cours.

\section{Le rôle central des professionnels dans l'encadrement pédagogique}

La plupart des formations sont dirigées par des universitaires, le plus souvent impliqués à titre individuel dans le secteur culturel, même si l'on trouve aussi des formations dirigées uniquement par un professionnel. Cependant, les formations codirigées par des universitaires et des professionnels sont assez courantes.

Bien souvent, les professionnels représentent de 50 à $75 \%$ des enseignants, voire davantage si l'on considère les heures dispensées. La relation avec les milieux professionnels est recherchée de façon constante et les postes de professeurs associés $\left(\mathrm{PAST}, \mathrm{PRAS}^{10}{ }^{10}\right.$ sont nombreux, mais la compétence

\footnotetext{
9. Les éléments qualitatifs repris ici proviennent de l'analyse réalisée durant la deuxième phase de l'étude auprès de 16 formations de niveau I, majoritairement universitaires (11 sur 16). Les exemples cités renvoient aux formations étudiées.

10. PAST, PRAS : professeurs associés ayant vocation à effectuer leur service sous forme de cours. Ils doivent justifier d'une expérience professionnelle autre qu'une activité d'enseignement en rapport avec la spécialité enseignée.
} 
pédagogique de ces professionnels est finalement peu interrogée. En corollaire, on relève une évolution des formations vers une «technicisation» de leurs enseignements en termes de contenus et de pédagogie (études de cas, travaux personnels, tutorat, développement de projets individuels et/ou collectifs...).

\section{La relative faiblesse de l'ouverture internationale}

Si les dimensions européenne et internationale sont citées comme une nécessité, elles sont encore peu développées dans les cursus et les partenariats malgré des exceptions parmi lesquelles on peut citer l'IEP de Grenoble, Paris VIII, l'Esc de Brest, le master de Lyon II/ARSEC, celui de La Rochelle qui s'appuie sur un réseau européen de villes...). On constate en effet que l'enseignement des langues - sauf l'anglais dans certaines formations - ne constitue pas une priorité, que le recrutement d'étudiants étrangers est peu important, que l'invitation de professeurs étrangers est rare et les relations avec des universités étrangères peu répandues ${ }^{11}$.

\section{Insertion professionnelle et perspectives d'emploi}

Selon les responsables interrogés, leurs formations produisent des résultats satisfaisants en matière d'insertion professionnelle et leurs filières répondent à de réels besoins. Toutefois, ce satisfecit est tempéré par certains qui déclarent que les conditions d'insertion des étudiants se traduisent par des rémunérations assez basses, par des contrats le plus souvent à durée déterminée et par une nécessaire mobilité. En fait, en l'absence d'études d'insertion et d'outils de suivi spécifiques et en raison de relations peu formalisées avec les diplômés, bien que les réseaux d'anciens étudiants soient encouragés de façon systématique, il est relativement difficile de connaître l'insertion professionnelle et les parcours des diplômés de ces formations.

\section{Des formations tournées vers le secteur privé pour les écoles de management}

Les écoles de management sont apparues récemment dans le champ de ces formations. Leur nombre, certes, est limité au regard de celui des formations universitaires mais ce phénomène suscite des questions sur les transformations du champ culturel qu'elles accompagnent.

Les écoles membres de la CGE proposent plusieurs types de formations de niveau I. Les neuf mastères spécialisés de niveau bac +6 , qui préparent à une forte spécialisation sectorielle ou à l'obtention d'une double compétence, sont destinés à des diplômés en fin de parcours et à des salariés en formation continue. Ils forment soit des généralistes de la gestion des organisations culturelles, soit des « spécialistes » dans une fonction ou une filière (directeur technique du spectacle vivant, édition, audiovisuel...). Pour être accréditées, ces formations sont analysées notamment en termes d'adéquation au marché de l'emploi auquel elles préparent.

\section{Des enseignements optionnels}

Outre ces mastères spécialisés, plusieurs écoles proposent à leurs étudiants en fin de cursus $(\mathrm{bac}+5)$ des enseignements de spécialisation (les «majeures», «mineures», « options », «électifs »...) dédiés aux secteurs culturels. Certaines, comme la «majeure culture»d'Hec, existent depuis plus de 20 ans. Dans le cadre de cette étude, 12 spécialisations de ce type ont été recensées, qui recouvrent des contenus et des volumes horaires extrêmement variés (60 heures pour l' «option» culture à l'Esc de Clermont-Ferrand, 135 heures pour la «majeure » de l'EDHEC à Lille, et 180 pour celle d'Hec). Par le biais de ces options, les étudiants issus d'un parcours en management classique sont initiés aux spécificités sectorielles, managériales de la culture, et parfois aux disciplines et problématiques artistiques. Dans les cursus de spécialisation proposés par ces écoles, les étudiants issus des formations universitaires (lettres, sciences sociales ou juridiques, filières artistiques) sont particulièrement nombreux.

\section{Des formations orientées vers le privé et l'international}

À entendre les responsables de ces formations, les formations universitaires préparent majoritairement aux métiers liés aux politiques publiques de la culture mais ne satisferaient pas systématiquement les besoins des organisations culturelles privées (marchandes ou non, subventionnées ou non), notamment en région. C'est pourquoi les formations consulaires recensées par l'étude orientent leurs étudiants vers les postes à pourvoir dans les

11. Les formations françaises sont d'ailleurs peu représentées au sein de l'European Network of Cultural Administration Training Centres (ENCATC). 
structures privées et associatives subventionnées (fonctions d'administrateurs, de chargés de diffusion ou de production, de responsables des partenariats privés...). L'ouverture internationale de ces formations permet en outre à leurs diplômés de postuler à des fonctions à l'étranger.

Les formations des écoles de management accompagneraient donc l'évolution d'une partie du secteur culturel - y compris dans le secteur subventionné - vers une logique de marché, de diffusion, de conquête des publics, de recherche de nouvelles voies de développement ou de financement. Sur ce plan, leur légitimité est de nature à rassurer étudiants et employeurs.

\section{DES FORMATIONS \\ ENTRE EUROPÉANISATION}

ET ANCRAGE RÉGIONAL

\section{Des formations inscrites dans l'espace européen}

Les systèmes d'enseignement supérieur s'européanisent, c'est là une des clés pour comprendre l'évolution que connaît le secteur des formations à l'administration et à la gestion de la culture. En effet, la construction d'un espace européen de l'enseignement supérieur, avec le processus de Bologne et la stratégie de Lisbonne mis en œuvre en 2000 (voir encadré page 3), a conduit les universités européennes à entrer dans un nouveau cycle de réformes dont la réforme LMD - licence-master-doctorat, respectivement en 3, 5 et 8 années d'études - n'est que la traduction au niveau national.

L'augmentation importante du nombre d'étudiants et l'avènement d'une université de masse dans les pays européens transforment considérablement le contexte dans lequel évoluent les formations à l'administration et à la gestion de la culture. Par ailleurs, si les études sur le management culturel et artistique attirent beaucoup d'étudiants, c'est aussi le cas en ce qui concerne les enseignants, car le secteur culturel est dynamique en Europe. Aussi, dès les années 1960, l'augmentation des effectifs étudiants et leur diversification ont amené partout en Europe la création de programmes d'études supérieures professionnalisants avec la mise en place d'institutions dont l'autonomie par rapport aux universités est plus ou moins grande ${ }^{12}$. Puis, ce furent les universités qui créèrent des DESS au cours de la décennie 1980, et des licences professionnelles à la fin des années 1990.

\section{La professionnalisation pour obligation...}

Que les universités soient confrontées à la nécessité de préparer les étudiants à l'entrée sur le marché du travail et non plus seulement dans les carrières académiques n'est pas un fait nouveau, mais le processus de Bologne les incite explicitement à développer ces nouvelles missions et à répondre à la question de l'employabilité de leurs étudiants. Cette évolution implique un développement et une diversification des filières de formation et une remise en cause de la centralité des formations doctorales. Elle nécessite une ouverture de l'Université en direction de la société, des entreprises et des collectivités territoriales ; une connaissance du marché du travail non seulement national mais également international et régional. Or, pour établir cette connaissance, il existe encore peu d'outils.

En 2006, l'ENCATC - réseau européen des formations à l'administration culturelle - a mené une étude sur l'impact de ce processus, laquelle soulignait l'ancienneté de certaines de ces formations (les premières se sont créées il y a plus d'une vingtaine d'années) et leur hétérogénéité. Si la majorité de ces formations sont délivrées dans les universités pluridisciplinaires et les polytechnics, elles le sont également dans les écoles artistiques, les écoles de commerce demeurant très minoritaires.

\section{... mais une Université encore peu en phase avec ce nouveau contexte}

Le développement important des formations de niveau master, non régulé au niveau national en France, apparaît comme un effet pervers du processus de Bologne. La perception par bon nombre de professeurs de «l'évidence» de formations liées à la culture dont l'Université est un lieu essentiel, ainsi que la nécessité dans laquelle ils sont de construire leur propre master, font que ces formations se développent dorénavant sans que leur relation au champ professionnel soit assurée, et entraînent un certain repli sur les enseignants universitaires au détriment de la participation des professionnels de la culture. Cette situation est donc paradoxale rapportée à la volonté de développer des formations professionnalisantes.

Autre symptôme d'une «focalisation universitaire » redoutée par certains: l'intégration des anciens DESS dans des diplômes d'abord discipli-

12. Parmi les institutions plutôt plus autonomes, citons les polytechnics en Grande-Bretagne ou en Finlande, Fachhochschule en Allemagne. Les IUT, en revanche, sont plutôt moins autonomes. 
naires, alors que le processus de Bologne aurait pu favoriser la création de nouveaux champs thématiques de recherche et de formation sur le management culturel. Il est sans doute prématuré de conclure quant à l'impact du processus de Bologne sur ces formations : cette réforme étant très récente, elle est encore susceptible de nombreuses évolutions.

\section{Des politiques de formation de plus en plus régionalisées}

L'investissement croissant des collectivités - en particulier des régions - en matière d'aménagement du territoire s'articule avec la politique conduite en ce domaine au niveau national ${ }^{13}$ et avec la politique régionale de l'Union européenne.

Les politiques en matière de formations universitaires dans le domaine culturel semblent, dans une certaine mesure, participer de cette territorialisation de l'aménagement du territoire - laquelle se situe parfois à rebours du processus de Bologne - et d'une politique nationale qui reste essentiellement fondée sur une logique de diplômes nationaux habilités par l'État et expertisés pour son compte.

\section{La formation, élément}

\section{de stratégie de développement économique pour les régions}

Les régions ont depuis longtemps investi le terrain de la formation professionnelle pour en faire un élément de leur stratégie de développement économique avec un triple objectif: répondre aux besoins de qualification des emplois régionaux, maintenir et accroître sur leurs territoires une offre de formation capable de satisfaire les populations régionales, voire attirer des étudiants, enseignants et chercheurs extérieurs. En outre, des moyens substantiels sont accordés par les régions aux établissements d'enseignement supérieur pour soutenir leurs besoins en équipements, développer la mobilité et les échanges d'étudiants et d'enseignants, favoriser les activités de recherche.

\section{Le triple enjeu de l'implication régionale}

Cette accentuation de l'implication régionale recouvre plusieurs enjeux importants pour une meilleure régulation de l'offre de formation au niveau régional mais également national.

Premier enjeu, celui d'amener ainsi les responsables de formation à accorder une attention plus grande à la situation de l'emploi culturel. Des interrogations peuvent naître à l'égard d'une régionalisation forcée des carrières même si $60 \%$ de l'emploi culturel se trouve déjà concentré dans les régions Île-de-France, Provence-Alpes-Côte d'Azur et Rhône- Alpes alors que l'impératif de mobilité s'impose dans les carrières culturelles.

Deuxième enjeu, corriger certains déséquilibres territoriaux en prenant en compte la situation régionale : en effet, le motif souvent avancé pour justifier la création d'un nouveau diplôme est qu'il n'y a pas de formations ou qu'elles sont rares. Cependant, l'ancrage disciplinaire des formations culturelles est d'une hétérogénéité telle que l'expertise effectuée au niveau national, à laquelle sont soumis les dossiers d'habilitation, interdit les rapprochements nécessaires à une régulation territoriale. Il faut ajouter à cela les compétitions internes au champ universitaire et les rivalités entre territoires (régions, agglomérations urbaines, départements) pour comprendre la limite de l'impact d'une volonté de planification régionale.

Troisième enjeu, organiser une coopération entre responsables de formations et milieux professionnels.

\section{TROIS OBJECTIFS : OBSERVER, ÉVALUER, RÉGULER}

Le contexte européen, l'évolution du marché de l'emploi culturel, le renouvellement des modes de financement de la culture et les politiques d'aménagement des territoires en cours sont autant de facteurs qui suggèrent de mieux observer, évaluer et réguler les formations à l'administration et à la gestion culturelles.

\section{Mieux connaître le marché}

Ce marché est porté par une dynamique de professionnalisation qui demeure fragile. L'absence de données spécifiques sur les emplois liés à l'administration et à la gestion relevant du secteur culturel tient à l'impossibilité de les isoler au sein des nomenclatures d'activités et de métiers utilisées par les enquêtes sur l'emploi culturel. En termes qualitatifs, il existe très peu de descriptifs fins de ces emplois. Les observatoires des organismes nationaux qui s'intéressent à cette question - le CNFPT et l'ANPE notamment - ne proposent que des élé-

13. En France, la politique d'aménagement du territoire est prise en charge notamment par la Délégation interministérielle à l'aménagement et à la compétitivité des territoires (DIACT). 
ments partiels d'appréciation : la filière culturelle de la fonction publique territoriale ne comprend que deux branches (patrimoine-bibliothèques et enseignement artistique), tandis que le Répertoire opérationnel des métiers et des emplois de l'ANPE donne peu de précisions sur les emplois de l'administration et de la gestion culturelle ${ }^{14}$.

Pourtant, la reconnaissance de l'importance de ces fonctions est au cœur des discours sur l'économie de la culture depuis les années 1980, et les politiques mises en place en faveur de l'aide à l'emploi et de la gestion des entreprises culturelles ont insufflé dans le secteur une logique entrepreneuriale de plus en plus prégnante.

En outre, si le dynamisme du secteur culturel repéré depuis deux décennies devait se poursuivre, il est probable que devraient encore se développer les emplois d'administration et de gestion, de même que ceux relatifs à la diffusion de la culture - métiers de la médiation - et au développement de la demande culturelle - métiers concernant le développement des publics. Le contenu de ces emplois devrait aussi évoluer sous l'effet du décloisonnement interne au secteur culturel, se traduisant par davantage de transdisciplinarité et de transversalité.

\section{Articuler les logiques de l'enseignement supérieur et du marché du travail}

Les responsables de formation, qui veulent évaluer l'insertion des étudiants sur le marché de l'emploi, recourent à des moyens assez informels et intuitifs. L'insertion fonctionne encore largement sur des principes de compagnonnage et de réseau malgré un infléchissement notable ces dernières années avec le développement des petites annonces, la mise en œuvre de prérequis en matière de diplômes, la rédaction de profils de poste, etc. Nombreux sont ceux qui, pour ajuster les contenus de formations aux besoins perçus du marché, développent une stratégie de veille empirique sous la forme de relations régulières avec le milieu professionnel.

Mais le processus de Bologne, dans lequel s'inscrit progressivement l'ensemble des formations supérieures, exige que les établissements d'enseignement supérieur et leurs enseignants mettent en place des outils de mesure de l'insertion professionnelle de leurs diplômés et d'évaluation de leurs formations. Cette exigence devrait permettre de cer- ner les spécificités de l'emploi culturel, notamment la mobilité constitutive des carrières. Or, remplir cette obligation d'évaluation est par ailleurs du ressort de chaque université, ce qui peut hypothéquer les possibilités de comparaisons et compromettre la capitalisation des connaissances.

Il faut également souligner que les processus d'habilitation des formations (fondés sur un rattachement disciplinaire et des pratiques proches de la cooptation) prennent peu en compte les logiques de métiers, l'utilité sociale réelle des formations et leur adéquation avec le marché du travail. Jusqu'à une date récente, n'étaient pas non plus envisagées de modalités d'évaluation des formations tant par les étudiants que par les pairs (notamment étrangers comme cela se pratique dans la recherche en matière d'évaluation des équipes et des projets).

\section{Rapprocher l'offre et la demande d'emploi}

L'avenir du secteur culturel ne peut être envisagé sans que soit trouvé le moyen d'articuler concrètement l'observation du marché de l'emploi et l'évaluation de l'insertion professionnelle des jeunes diplômés et, à partir de là, d'en tirer des conséquences en matière de régulation des formations supérieures. Plusieurs pistes sont ouvertes pour favoriser la mise en place d'une connaissance partagée des réalités professionnelles et de l'évolution des formations, dans le contexte d'un marché du travail en permanente évolution, parmi lesquelles : - l'identification des compétences à maîtriser dans l'exercice des emplois conduit à la notion de « référentiel de métiers ou d'activités », peu connue et peu pratiquée par les enseignants-chercheurs sauf dans certains secteurs ciblés (par exemple les IUT ou les BTS). Cette approche est en train d'être engagée sous plusieurs formes ;

- l'intégration potentielle de l'ensemble des diplômes dans le Répertoire national des certifications professionnelles $\left(\mathrm{RNCP}^{15}\right)$ constitue une bonne occasion d'introduire progressivement des éléments d'une approche «métiers »;

- la réalisation par la Commission paritaire nationale emploi-formation du spectacle vivant (CPNEF-SV) d'un guide des métiers du spectacle vivant avec la rédaction de référentiels, ainsi que d'un répertoire des formations certifiantes ou

14. La base d'emplois Cortex (cortex-culturemploi.com) apporte, pour sa part, des indications sur les besoins des employeurs et les demandeurs d'emploi.

15. Chaque diplôme, même inscrit de droit comme par exemple les diplômes relevant de l'enseignement supérieur, est inscrit sous la forme d'une « fiche Répertoire » fournissant notamment le « résumé du référentiel d'emploi ou éléments de compétence acquis ». 
diplômantes identifiant les emplois visés par les formations en partenariat avec le ministère de la Culture et de la Communication (DMDTS, DDAI). Ce partenariat a également contribué à mettre en place une commission professionnelle consultative (CPC) pour le spectacle vivant chargée d'analyser l'offre de formation du ministère au regard de l'emploi. Des chantiers analogues sont poursuivis par l'Observatoire prospectif des métiers et des qualifications au sein de la CPNEF-SV. Dans le même esprit, des travaux devraient être engagés sur les référentiels métiers du CNFPT;

- la création des contrats d'objectifs emploi-formation (COEF), qui réunit des représentants des ministères concernés (Culture, Emploi), des syndicats professionnels (patronaux et salariés) et des conseils régionaux.
$* * *$

L'administration et la gestion des entreprises et institutions culturelles constituent un segment très spécifique de l'emploi culturel en même temps qu'elles font partie du vaste ensemble des métiers du management. Elles se trouvent ainsi aux confins de ces deux champs, là où se rencontrent les logiques propres à chacun d'eux. Leurs professionnels sont quotidiennement confrontés au défi qui consiste à concilier exigences artistiques et culturelles et dynamiques économiques. Au-delà des nécessaires ajustements entre l'offre de formation et les besoins du secteur, les formations qui se positionnent sur ce segment se doivent d'intégrer cette spécificité et de faire saisir toute la complexité intrinsèque de ces métiers.

\section{RÉSUMÉ}

Les formations à la gestion et à l'administration culturelles se sont multipliées en France depuis les années 1980 avec le développement du secteur culturel, la professionnalisation de l'emploi dans ce secteur mais aussi l'attrait pour la culture en général. Ce sont près de 300 formations, majoritairement de niveau I sous forme de masters professionnels, qui ont pu être répertoriées et caractérisées (ancienneté, nombre d'étudiants, domaine d'application et, le cas échéant, champ disciplinaire, types d'enseignement). Ces formations sont essentiellement universitaires même si des écoles de commerce consulaires ont pris position plus récemment sur ce marché. Cette explosion du nombre de formations doit être resituée dans un double contexte : celui d'un espace européen des formations initié par le processus de Bologne et la réforme LMD, qui semblent avoir contribué à la multiplication de ces formations au sein de l'Université ; celui de la territorialisation des politiques de formation et d'une implication accrue du niveau régional. L'intérêt du secteur culturel comme des étudiants attirés par ces formations est que ce marché de l'emploi de gestion et d'administration culturelles soit à la fois mieux apprécié quantitativement et mieux suivi qualitativement, et que les logiques respectives de l'enseignement supérieur et du marché de l'emploi se confrontent dans des pratiques régulières d'évaluation, permettant ainsi de rapprocher offre et demande d'emploi dans une véritable démarche de régulation.

\section{ABSTRACT}

In France, since the 1980s, training in cultural management and administration has greatly expanded, in step with the cultural sector's growth and increasing professionalisation, and the public's liking for culture in general. Some three hundred courses, mostly level one (professional masterships), have been listed and described - their time in operation, number of trainees, field covered, subject area where relevant, nature of instruction. For the most part, training is associated with universities, although decentralised business schools have recently begun to enter the market. The surge in training courses needs to be viewed against a two-fold background: first, a European training context issuing from the Bologna process accompanied by the 'BMD'(Bachelors, Masters, Doctorates) educational reform which appears to have stimulated a proliferation of such cultural courses in the universities; second, a territorialisation of training policies entailing greater regional involvement. The interest of the cultural sector, as of the students drawn to this type of training, lies in the fact that the cultural management and administration job market has become more highly valued quantitatively and better ordained qualitatively. Moreover, the respective goals of higher education and the labour market meet together through a regular process of evaluation. This form of regulation enables labour supply more closely to match demand. 